Pacific Journal of Mathematics

THOSE ABELIAN GROUPS CHARACTERIZED BY THEIR
COMPLETELY DECOMPOSABLE SUBGROUPS OF FINITE
RANK

WU-Chung HSIANG AND WU-YI HSIANC 


\title{
THOSE ABELIAN GROUPS CHARACTERIZED BY THEIR COMPLETELY DECOMPOSABLE SUBGROUPS OF FINITE RANK
}

\author{
Wu-ChUNG HSIANG* AND Wu-YI HSIANG \\ (To The Memory of Our Grandparents)
}

I. A non-zero group ${ }^{1}$ is said to have rank $n$, if any finitely generated subgroup can be generated by $n$ or fewer elements and $n$ is the smallest integer with this property. ${ }^{2}$ A group $G$ is completely decomposable if $G$ is a direct sum of groups of rank one. ${ }^{3}$ A subgroup $H$ of a given group $G$ is completely reducible in $G$ if there exists a direct decomposition of $G$

$$
G=\Sigma_{j} G_{j}+G^{\prime}
$$

such that all $G_{j}$ 's are of rank one and

$$
H=\Sigma_{\jmath}\left(H \cap G_{j}\right) .
$$

Roughly speaking, a subgroup $H$ is a completely reducible subgroup of $G$ if it is a direct sum of rank one groups in a way that can be pulled up to a decomposition of $G$. It is clear that every completely reducible subgroup $H$ of $G$ is completely decomposable. But in general, the converse is not true. The main purpose of this paper is to determine those groups in which complete reducibility coincides with the complete decomposability for some classes of subgroups.

For convenience, the following terminology is adopted.

Definition 1.1. A group is said to have property $(\alpha)$, if every subgroup of rank one is completely reducible in it.

Definition 1.2. A group is said to have property $(\beta)$ if every completely decomposable subgroup of finite rank is completely reducible in it.

Definition 1.3. A group is said to have property $(\gamma)$ if every subgroup of finite rank is completely reducible in it.

Received March 9, 1960. This paper was prepared when the first author held a predoctoral fellowship at Princeton University and the second author was in the military service in Formosa.

1 In this paper, "group" will always mean "abelian group".

2 This definition of rank was given in [5].

${ }^{3}$ All the groups of rank one are known to us; e.g., a primary group of rank one is either cyclic or of the type $Z\left(p^{\infty}\right)$ and a torsion-free group of rank one is a subgroup of the additive group of all rational numbers. In torsion-free case, the above definition for completely decomposable groups coincides with the usual one; e.g., see [4 pp. 211-216]. 
It follows from the definitions that property $(\gamma)$ implies property $(\beta)$ and property $(\beta)$ implies property $(\alpha)$.

We begin with the following lemmas dealing with those torsion groups having the above properties.

LEMMA 1.1. A torsion group $G$ has property $(\alpha),(\beta)$ or $(\gamma)$ if and only if each of its primary components has property $(\alpha),(\beta)$ or $(\gamma)$ respectively.

Proof. We decompose $G$ into its $p$-primary components

$$
G=\Sigma_{p} G^{(p)} .
$$

Suppose $G$ has property $(\beta)$. Let $H^{(p)}$ be a completely decomposable subgroup of finite rank in the $p$-primary component $G^{(p)}$ of $G$. By our assumption that $G$ has property $(\beta)$, we have a direct decomposition of $G$

$$
G=\Sigma_{j} G_{j}+G^{\prime}
$$

satisfying the following conditions:

(i) each $G$, has rank one

(ii) $H^{(p)}=\Sigma_{j}\left(G_{j} \cap H^{(p)}\right)$.

Since $H^{(p)}$ is contained in the $p$-primary component $G^{(p)}$ of $G$, we get what we need by simply considering the $p$-primary components of the decomposition (4).

Conversely, suppose that each $G^{(p)}$ had property $(\beta)$. Let $H$ be a given completely decomposable subgroup of finite rank $n$ in $G$. It is clear that each $p$-primary component $H^{(p)}$ of $H$ is either zero or also completely decomposable. Since we suppose that each $G^{(p)}$ has property $(\beta)$, there exists a direct decomposition of $G^{(p)}$ for each $p$

$$
G^{(p)}=\sum_{j=1}^{n_{p}}\left(G_{j}^{(p)} \cap H^{(p)}\right)
$$

satisfying the following conditions:

(i) each $G_{j}^{(p)}$ is at most of rank one,

(ii) $H^{(p)}=\sum_{j=1}^{n_{p}}\left(G_{j}^{(p)} \cap H^{(p)}\right)$

(iii) $0 \leqq n_{p} \leqq n$

(iv) the maximum of $n_{p}$ is $n$.

One may make $n_{p}=n$ by inserting zero groups. So, we assume $n_{p}=n$ for each $p$.

Denote $\Sigma_{p} G_{j}^{(p)}$ by $G_{j}$ and $\Sigma_{p} G_{p}^{\prime}$ by $G^{\prime}$. It is easy to see that each $G_{j}$ has rank one and

$$
G=\sum_{j=1}^{n} G_{j}+G^{\prime}, \quad H=\sum_{j=1}^{n}\left(H \cap G_{j}\right) .
$$

This means that $G$ has property $(\beta)$. The other two parts of the lemma 
can be shown in an analogous manner.

Lemma 1.2. If a torsion group has property $(\alpha)$, then each of its p-primary components is either divisible or of the form

$$
\Sigma_{j_{p}} z_{j_{p}}\left(p^{h_{p}}\right)+\Sigma_{k_{p}} z_{k_{p}}\left(p^{h_{p+1}}\right) .^{4}
$$

Conversely, if each p-primary component of a torsion group $G$ is either divisible or of the form (7), then $G$ has property $(\gamma)$.

Proof. It suffices to show that a $p$-primary groups having property $(\alpha)$ must be either divisible or reduced, since all the other cases are sonsequences of Lemma 1.1 and some known results [3, Lemma III]. If this is not the case, then we can find a group $G$ with property $(\alpha)$ of the following form

$$
Z\left(p^{h}\right)+Z\left(p^{\infty}\right)+G^{\prime}
$$

where $Z\left(p^{h}\right)$ is a cyclic group of order $p^{h}$ generated by $u_{1}$ and is a $p$-primary divisible group of rank one. Let $u_{2}$ be an element in $Z\left(p^{\infty}\right)$ of order $p^{h+1}$. Consider the subgroup $U$ generated by $u_{1}+u_{2}$. Since $G$ has property $(\alpha)$, there exists a direct decomposition of $G$

$$
G=H+K
$$

such that $U \subset H$ and $H$ is of rank one. It is clear that the $p$-layer ${ }^{5}$ of $H$ coincides with that of $U$, and it is a cyclic group generated by $p^{h} u_{2}$. Since $H$ is a direct summand of $G$, the heights of its elements in it are equal to the heights of those in $G$. It follows that $p^{h} u_{2}$ is of infinite height in $H$ and $H$ is divisible. Since $u_{1}+u_{2} \in U \subset H, u_{1}+u_{2}$ is of infinite height in $G$. On the other hand, it follows from (8) that the height of $u_{1}+u_{2}$ is equal to the minimum of the heights of $u_{1}$ and $u_{2}$ in $G$. Since the minimum is 0 , we have a contradiction. This completes our proof.

II. Groups with property $(\alpha)$. To discuss groups with property $(\alpha)$, we need the following lemmas.

Lemma 2.1. If a group $G$ has property $(\alpha)$, so does its torsion subgroup $T(G)$.

It is an immediate consequence.

Lemma 2.2. A torsion-free group $G$ has property $(\alpha)$ if and only if (a) $G$ and $G / D$ are separable ${ }^{6}$, where $D$ is the maximal divisible

$4 Z(e)$ denotes the cyclic group of order $e$.

5 The $p$-layer of a primary group $G$ is defined to be the subgroup $P=\{x \mid p x=0, x \in G\}$.

${ }^{6}$ A torsion-free group is called separable, if every finite subset is contained in a completely decomposable direct summand (See $[\mathbf{1}]$ ). 
subgroup of $G$,

(b) all the elements $\neq 0$ in $G / D$ have the same type [4, pp. 207-211] in $G / D$.

Proof. In fact, it is known [1, Corollary 4.5] that a torsion-free group $G$ satisfies conditions (a) and (b) if and only if every pure subgroup of finite rank is a direct summand. Obviously, the property that every pure subgroup of finite rank is a direct summand implies our property $(\alpha)$ which deals with subgroup of rank one. We prove the converse by finite induction on the rank of the pure subgroups in $G$. It is trivially true for the pure subgroups of rank one in $G$. Now, let $H$ be a pure subgroup of rank $n$ and $\left\{x_{1}, x_{2}, \cdots, x_{n-1}, x_{n}\right\}$ be one of its maximal independent subsets. By induction hypothesis, $G$ can be expressed as

$$
G_{1}+G_{2}
$$

where $G_{1}$ is the pure subgroup generated by $\left\{x_{1}, x_{2}, \cdots, x_{n-1}\right\}$. Then $x_{n}$ can be expressed as

$$
y_{1}+y_{2}
$$

where $y_{i} \in G_{i}(i=1,2)$ and $y_{2} \neq 0$. Since $G$ has property $(\alpha)$, the pure subgroup $Y$ generated by $y_{2}$ is a direct summand of $G_{2}$. Consequently, $H=G_{1}+Y$ is a direct summand of $G$.

From now on, a torsion-free group satisfying the conditions (a) and (b) in Lemma 2.2 will be called a uniformly separable group.

In fact, one can show that every reduced uniformly separable group is isomorphic to a subgroup of a strong direct sum of torsion-free groups of rank one each of which has the same type ${ }^{7}$ as $G$.

Lemma 2.3. If $G$ has property $(\alpha)$ and $T(G)$ is divisible, then $G / T(G)$ has property $(\alpha)$.

Proof. Let $U$ be a rank one subgroup if $G / T(G)$. Consider the complete inverse image $\widetilde{U}$ of $U$ with respect to the natural projection $q: G \rightarrow G / T(G)$. Since $T(G)$ is divisible, we have $\widetilde{U}=U_{1}+T(G)$ where $U_{1}$ is a rank one torsion-free subgroup in $G$. Applying the presumed property $(\alpha)$ in $G$ and passing to the natural projection, we have our property $(\alpha)$ in $G / T(G)$.

THEOREM A. A group $G$ has property $(\alpha)$ if and only if

(a) $G$ is a torsion group with property $(\alpha)$ (see Lemma 1.2), or

7 In a reduced uniformly separable group, all the non-zero elements have the same type; "the type of $G$ " therefore has a definite meaning. 
(b) $G=J+D$ where $J$ is a reduced uniformly separable group and $D$ is divisible.

Proof. Suppose $G$ has property $(\alpha)$. If the torsion subgroup $T(G)$ of $G$ is divisible, then $G=J_{0}+T(G)$ where $J_{0}$ is torsion-free and $J_{0}$ has property $(\alpha)$ by Lemma 2.3. Now by Lemma 2.2, and hence $G$ has form (b). We now show that if $T(G)$ is not divisible, then $T(G)=G$, so that $G$ has form (a). If this is not the case, we can find a group $G$ with property $(\alpha)$ of the following form

$$
R_{1}+T^{\left(p_{1}\right)}(G)+G^{\prime 8}
$$

where $R_{1}$ is a torsion-free group of rank one and $T^{\left(p_{1}\right)}(G)$ is a non-zero $p_{1}$-primary component of the torsion subgroup $T(G)$ which, by assumption, is of the form (b). There are two cases according to the type $\left|R_{1}\right|$ of $R_{1}{ }^{9}$

Case 1. The type $\left|R_{1}\right|$ has value $\infty$ at $p_{1}$. In this case, we consider the cyclic subgroup $\widetilde{R}$ generated by $r_{1}+a_{p_{1}}$ where and $a_{p_{1}}$ are non-zero elements in $R_{1}$ and $T^{\left(p_{1}\right)}(G)$ respectively. Since $G$ has property $(\alpha)$ and $R$ is a torsion-free subgroup of rank one in $G$, we can decompose $G$ as

$$
R_{2}+T^{\left(p_{1}\right)}(G)+T^{\prime \prime}
$$

such that (i) $R_{2}$ is a torsion-free direct summand of rank one in $G$ and (ii) $\widetilde{R} \subset R_{2}$. It follows from (13ii) that $p^{h_{p_{1}+1}} r_{1}\left(=p_{1}^{h_{p_{1}+1}}\left(r_{1}+a_{p_{1}}\right)\right)$ whose type has value $\infty$ at $p_{1}$, is an element in $R_{2}$. Hence $\left|R_{2}\right|$ has value $\infty$ at $p_{1}$. On the other hand, it also follows from (13ii) that $r_{1}+a_{p_{1}}$, which is not divisible by $p_{1}^{h_{1} p_{1}+2}$, is an element of $R_{2}$ also. This means $\left|R_{2}\right|$ cannot have the value $\infty$ at $p_{1}$, hence, we have a contradiction. Thus, Case 1 cannot exist.

Case 2. The type $\left|R_{1}\right|$ has a finite value at $p_{1}$. In this case, we consider a torsion-free cyclic subgroup $U$ of $G$ satisfying the following conditions:

(i) $U$ is generated by $p_{1}^{s} r_{1}+a_{p_{1}}$ where $r_{1} \in R_{1}$ and $a_{p_{1}} \in T^{\left(p_{1}\right)}(G)$

(ii) the characteristic [4, pp. 207-211] of $r_{1}$ in $R_{1}$ has zero value at $p_{1}$.

(iii) $s>h_{p_{1}}+1 \geqq 1$.

Since $G$ has property $(\alpha)$, we have the following decomposition

${ }^{8}$ Here, we use the fact that every $p$-primary subgroup of bounded order is a direct summand.

${ }^{9}$ A torsion-free group of rank one is automatically a uniformly separable group and all its elements have the same type. We denote this definite type by $|R|$. In fact, a torsion-free group of rank one is characterized by the type. 


$$
G=R_{3}+G^{\prime \prime}
$$

where $R_{3}$ is a torsion-free group of rank one and contains $U$ as a subgroup. It follows from our construction that $p_{1}^{s} r_{1}+a_{p_{1}}$ is not divisible by $p_{1}$ in $G$. Consider the factor group $G / T(G)$; we have

$$
R_{1}+\left(T^{\left(p_{1}\right)}(G)+G^{\prime}\right) / T(G)=G / T(G)=R_{3}+G^{\prime \prime} / T(G) .
$$

Since $p_{1}^{s} r_{1} \in R_{1}$, the characteristic of $p_{1}^{s} r_{1}+T(G)$ in $G / T(G)$ has value $s(>0)$ at $p_{1}$. On the other hand, since $p_{1}^{s} r_{1}+a_{p_{1}} \in U \subset R_{3}$, the characteristic of $\left(p_{1}^{s} r_{1}+a_{p_{1}}\right)+T(G)$ in $G / T(G)$ has value zero at $p_{1}$. But $\left(p_{1}^{s} r_{1}+a_{p_{1}}\right)+T(G)$ and $p_{1}^{s} r_{1}+T(G)$ represent the same element in $G / T(G)$. This means that Case 2 cannot exist either. Hence, the necessity is thereby proved.

Conversely, we prove the sufficiency; i.e., groups of form (a) or (b) have property $(\alpha)$. Groups of the form (a) have property $(\alpha)$. In the case of groups having the form (b), the torsion subgroup $T(G)$ is contained in $D$, which is divisible. Therefore $T(G)$ is divisible. Hence we have no trouble with those subgroups of rank one contained in $T(G)$. Now, we need only to prove that a torsion-free subgroup $L$ of rank one in $G$ can be enlarged to a direct summand of rank one in $G$. Let us consider the factor group $G / T(G)$ which satisfies the conditions (a) and (b) in Lemma 2.2. Therefore, $G / T(G)$ has property $(\alpha)$. Since $(L+T(G)) / T(G)$ is a torsion-free subgroup of rank one in $G / T(G)$, we can enlarge $(L+T(G)) / T(G)$ to a torsion-free direct summand $L$ of rank one in $G / T(G)$. Denote the complete inverse image of $L$ with respect to the natural projection $q: G \rightarrow G / T(G)$ by $S$. It is clear that $L \subset S$ and $L \cap T(G)=0$. By Zorn's Lemma, there exists a maximal subgroup $M$ of $S$ such that (i) $L \subset M$ and (ii) $M \cap T(G)=0$. It follows from the divisibility of $T(G)$ that $M$ is a direct summand of rank one in $S$. Moreover, since $(M+T(G)) / T(G)$ is a direct summand of $G / T(G)$ and $M \cap T(G)=0, M$ is in turn a direct summand of rank one in $G$. By our construction, $L \subset M$. We thereby complete our proof.

III. Groups with property $(\beta)$. As we did in $\S$ II, we first prove the following lemma.

Lemma 3.1. A torsion-free group $G$ has property $(\beta)$ if and only if it can be expressed as one of the following forms

(a) $J_{0}+D$ where $J_{0}$ is uniformly separable group of null type [4,"pp. 207-211] and $D$ is a divisible group.

(b) A torsion-free group of rank one.

Proof. Sufficiency: It is obvious that groups of the form (b) have property $(\beta)$. Let us turn to the case (a). We first recall that $J_{0}$ has 
the following properties:

(i) every subgroup of finite rank is free,

(ii) every pure subgroup of finite rank is a direct summand [1]. Now, let $H$ be a given completely decomposable subgroup of finite rank in $G$. We first consider the factor group $H /(D \cap H)$ where $D$ is the maximal divisible subgroup of $G$; by (i), we can directly decompose $H$ as

$$
H_{1}+(H \cap D)
$$

where $H_{1}$ is a free group of finite rank and $(H \cap D)$ is a completely decomposable subgroup of finite rank. Applying Zorn's lemma and divisibility of $D$ as before, $G$ can be directly decomposed as

$$
J_{0}^{\prime}+D
$$

such that

(i) $D$ has the same meeting as above,

(ii) $J_{0}^{\prime} \cong J_{0}$, and

(iii) $J_{0}^{\prime} \supset H_{1}$. It follows that the properties (i) and (ii) of $J_{0}$ that $H_{1}$ is completely reducible in $J_{0}^{\prime}$. To sum these results, $H$ is completely reducible in $G$.

Necessity. Now, we prove that a torsion-free group $G$ having property $(\beta)$ must be either (a) or (b). First, it follows from Lemma 2.2 that $G$ must be a uniformly separable group. If $G$ is neither of the form (a) nor of the form (b), we have a direct decomposition of $G$ as follows

$$
R_{1}+R_{2}+G^{\prime}
$$

such that

(i) both $R_{1}$ and $R_{2}$ are of rank one,

(ii) $\left|R_{2}\right|$ has finite value at some prime number $p_{0}$, and

(iii) $\left|R_{1}\right| \geqq\left|R_{2}\right|>$ the null type. By (18.ii), there exist $r_{i} \in R_{i}$ $(i=1,2)$ such that $r_{2}$ is not divisible by $p_{0}$ in $G$. Consider the subgroup $H=\widetilde{R}_{1}+R_{2}$ where $\widetilde{R}_{1}$ is the cyclic subgroup generated by $p_{0} r_{1}+r_{2}$ of rank two in it. There exists a direct decomposition of $G$ as follows

$$
G_{1}+G_{2}+G^{\prime \prime}
$$

such that

(i) $G_{1}$ and $G_{2}$ are of rank one, and

(ii) $H=\left(H \cap G_{1}\right)+\left(H \cap G_{2}\right)$. From (18. iii) and the fact that $R_{1}$ is of null type, we conclude that the set $\left\{\left(G_{1} \cap H\right),\left(G_{2} \cap H\right)\right\}$ of subgroups in $H$ coincides with $\left\{\widetilde{R}_{1}, R_{2}\right\}$ [1]. Say, $\widetilde{R}_{1}=G_{1} \cap H$ and $R_{2}=G_{2} \cap H$. Hence, $G_{1}$ and $G_{2}$ are the pure subgroups spanned by $p_{0} r_{1}+r_{2}$ and $r_{2}$ 
respectively. Since $\left(G_{1}+G_{2}\right)$ is a direct summand of $G$ and neither $r_{2}$ nor $p_{0} r_{1}+r_{2}$ is divisible by $p_{0}$ it follows from (19) that $p_{0} r_{1}=\left(p_{0} r_{1}+r_{2}\right)-r_{2}$ is not divisible by $p_{0}$ in $G$ either. We have a contradiction. Thus, $G$ must satisfy either (a) or (b).

LEMMA 3.2. Suppose that the torsion subgroup $T(G)$ of $G$ is divisible. Then, $G$ has property $(\beta)$ if and only if $G / T(G)$ does.

Proof. Necessity: Suppose that $G$ has property $(\beta)$. Let $H$ be a completely decomposable subgroup of finite rank in $G / T(G)$. Denote the complete inverse image of $H$ with respect to the natural projection $q: G \rightarrow G / T(G)$ by $S$. Since $S$ is divisible, we have

$$
S=\tilde{H}+T(G) .
$$

Clearly, $\widetilde{H}$ is isomorphic to $H$ and hence is a completely decomposable subgroup of finite rank in $G$. By our assumption that $G$ has property $(\beta), \widetilde{H}$ is completely reducible in $G$. Carrying back to the factor group $G / T(G)$, therefore, $H$ is completely reducible in $G / T(G)$.

Sufficiency: There are two cases according to whether $G / T(G)$ is of the form (b) or (a) in Lemma 3.1.

Case 1. $G / T(G)$ is a torsion-free group of rank one. Then, a completely decomposable subgroup $H$ of finite rank in $G$ can be expressed as

$$
R_{1}+(T(G) \cap H)
$$

where $R_{1}$ is the zero group or a torsion-free group of rank one. Since $T(G) \cap H=T(H)$ is a completely decomposable subgroup of $T(G)$ and since $T(G)$ is divisible, it follows from Lemma 1.2 that $(T(G) \cap H)$ is completely reducible in $T(G)$. On the other hand by applying Zorn's lemma and the divisibility of $T(G)$ as we did before, there exists a direct decomposition of $G$ as follows

$$
M+T(G)
$$

such that $M \supset R_{1}$. To sum up the above results we have proven the complete reducibility of $H$ in $G$.

Case 2. Suppose that $G$ has the form (a) in Lemma 3.1. Let $H$ be a completely decomposable subgroup of finite rank in $G$. We first consider the factor group $H /(H \cap D)$ where $D$ is the maximal divisible subgroup of $G$. Since $H /(H \cap D)$ is isomorphic to a free subgroup $(H+D) / D$ of finite rank in $G / D$, we can directly decompose $H$ as 


$$
H_{1}+(H \cap D)
$$

where $H_{1}=H /(H \cap D)$ is a free group of finite rank. It is clear that $(H \cap D)$ is also completely decomposable, since it is a direct summand of a completely decomposable group of finite rank. Hence, we have a refinement of (23) as follows

$$
H=H_{1}+D_{1}+(H \cap T(G))
$$

where $D_{1}+(H \cap T(G))=H \cap D$. Indeed, all the three terms on the right side of (24) are completely decomposable. Successively applying Zorn's lemma and divisibility of $D$ and $T(G)$, we can directly decompose $G$ as

$$
\widetilde{J}_{0}+\widetilde{D}+T(G)
$$

such that

(i) $\widetilde{D}+T(G)=D$ and $\widetilde{D} \supset D_{1}$, and

(ii) $\widetilde{J}_{0}$ is a uniformly separable of null type and $J_{0} \supset H_{1}$. It follows from Lemma 1.2 and Lemma 3.1 that $(H \cap T(G))$ and $\left(H_{1}+D_{1}\right)$ are completely reducible in $T(G)$ and $\left(\widetilde{J}_{0}+\widetilde{D}\right)$ respectively. To sum up these results, we have proven that $H$ is also completely reducible in $G$ in this case.

As a consequence of Theorem A, Lemmas 3.1 and 3.2, we have the following theorem

Theorem B. A group $G$ has property $(\beta)$ if and only if it can be expressed in one of the following forms:

(a) $J_{0}+D$ where $J_{0}$ is a uniformly separable group of null type and $D$ is a divisible group.

(b) $R+C$ where $R$ is a torsion-free group of rank one and $C$ is a torsion divisible group.

(c) $G$ is a torsion group with property $(\beta)$ (see Lemma 1.2).

REMARK. If we put $(\beta)$ in a stronger form $\left(\beta^{\prime}\right)$ every completely decomposable subgroup of $G$ is completely reducible in $G$, then (a) becomes

$\left(a^{\prime}\right) \quad F+D$ where $F$ is a free group of finite rank and $D$ is divisible.

IV. Group with property $(\gamma)$. We first find the torsion-free groups having property $(\gamma)$.

LEMMA 4.1. A torsion-free group has property $(\gamma)$ if and only if it can be expressed in one of the following forms

(a) $J_{0}+A$ where $J_{0}$ is a uniformly separable group of null type and $A$ is either the zero group of the additive group of all rational. 
numbers.

(b) A torsion-free group of rank one.

Proof. Necessity: It follows from Theorem B that a torsion-free group $G$ having property $(\gamma)$ is either of rank one or of the form

$$
J_{0}+D
$$

where $J_{0}$ is a uniformly separable group of null type and $D$ is a torsionfree divisible group. If it is the latter case, we claim that the rank of $D$ is at most one. For any torsion-free divisible group of rank greater than one contains an indecomposable subgroup of rank two, and such a subgroup is not completely reducible in $G$.

Sufficiency. We have no trouble with groups of the form (b). Suppose that $G$ is a group of the form (a). Let $H$ be a subgroup of finite rank in $G$. Consider the factor group $H / H \cap A$. Since $H / H \cap A$ is isomorphic to a subgroup of finite rank in $J_{0}$, we can directly decompose $H$ as

$$
F+H \cap A
$$

where $F$ is a free group of finite rank and $H \cap A$ is at most of rank one. This means that $H$ is completely decomposable. Finally, it follows from Theorem B that $H$ is completely reducible in $G$. The proof is thereby completed.

THEOREM C. A group has property $(\gamma)$ if and only if it can be expressed in one of the following forms:

(a) $J_{0}+A+\sum_{j=1}^{n} C_{p_{j}}$ where $J_{0}$ is a reduced uniformly separable group of null type. $A$ is either the zero group or the additive group

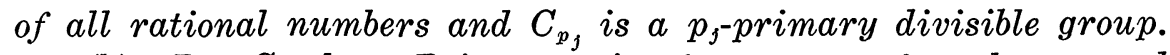

(b) $R+C$ where $R$ is a torsion-free group of rank one, and $C$ is a torsion divisible group. Moreover, there are only a finite number of primes $p$ such that the p-primary component of $C$ and the p-value of the type $|R|$ are simultaneously not zero.

(c) $J_{0}+C$ where $J_{0}$ is a reduced uniformly separable group of null type and $C$ is a torsion divisible group.

(d) $G$ is a torsion group with property $(\gamma)$ (See Lemma 1.2).

Proof. Necessity: It follows from Theorem B and Lemma 1.2 that $G$ is either of the form (d) or there exists a subgroup in the following form

$$
R+C
$$


$N$ here $R$ is a torsion-free group of rank one and $C$ is a torsion divisible group. We claim that in the latter case there are only a finite number of primes such that both the p-primary component of $C$ and the value of $|R|$ at $p$ are simultaneously not zero. If this is not the case, there exists an element $e$ in $R$ and a subgroup $E$ of $C$ satisfying the following conditions:

(i) $E$ is of the form

$$
\sum_{i} V\left(p_{i}\right)
$$$$
(i=1,2, \cdots)
$$

where $V\left(p_{i}\right)$ is a cyclic group of order $p_{i}$.

(ii) the equation

$$
p_{i} x=e
$$$$
(i=1,2, \cdots)
$$

is always solvable in $G$.

Let $\widetilde{e}\left(p_{i}\right)$ be the solution of $(30)$ and $\tilde{f}\left(p_{i}\right)$ be a non-zero element of $V\left(p_{i}\right)$ for $i=1,2, \cdots$. Since $C$ is divisible, there exists an element $f\left(p_{i}\right)$ for each $i$ such that $p_{i} f\left(p_{i}\right)=\widetilde{f}\left(p_{i}\right)$. Set $e\left(p_{i}\right)=\widetilde{e}\left(p_{i}\right)+f\left(p_{i}\right)$. Let $H$ be a subgroup of rank two in $G$ generated $E, e\left(p_{i}\right)$ 's, and $e . H$ is not completely reducible in $G$. In fact $H$ is not completely decomposable [2]. Therefore, a group having this property must be in one of the stated forms.

Sufficiency. In fact, we have already known that a group $G$ in one of those forms has property $(\beta)$. Therefore, to prove the theorem it sufficies to show that every subgroup $H$ of finite rank is always completely decomposable. There is no trouble with the groups of the form (d). In other cases, it is known [2] that $H$ can be directly decomposed as

$$
H_{1}+T(H)=H_{1}+H \cap T(H)
$$

where $T(H)$ is the torsion subgroup of $H$ and $H_{1}$ is isomorphic to a subgroup of $J_{0}+A, R$, or $J$ depending on which of cases (a), (b) or (c) holds. In any case, it follows from Lemma 4.1 that $H_{1}$ is completely decomposable. On the other hand, it is obvious that $T(H)$ is completely decomposable. The proof is thereby completed.

REMARK. If we put $(\gamma)$ in a stronger form $\left(\gamma^{\prime}\right)$ as follows: A group $G$ is said to have property $\left(\gamma^{\prime}\right)$ if every subgroup of $G$ is completely reducible in $G$; then Theorem $\mathrm{C}$ becomes Theorem $\mathrm{C}^{\prime}$ : A group $G$ has property $\left(\gamma^{\prime}\right)$ if and only if it can be expressed in one of the following forms

(a') $F+A+\sum_{j=1}^{n} C_{p_{i}}$ where $F$ is a free group of finite rank and $A, C_{p_{i}}$ have the same meaning as in (a).

(b') $R+C_{f}$ where $R$ has the same meaning as in (b) and $C_{f}$ is a 
torsion divisible group of finite rank. Moreover, the $p$-primary components of $C_{f}$ and $R$ subjected to the same relation as in (b).

$\left(c^{\prime}\right) \quad F+C_{f}$ where $F$ and $C_{f}$ have the same meaning as above.

The authors acknowledge with great pleasure their gratitude to. Professor Ross Beaumont and Professor David Harrison whose criticisms. and comments resulted in substantial improvements.

\section{REFERENCES}

1. R. Baer, Abelian groups without elements of finite order, Duke Math. J., 3 (1937), 68122.

2. The subgroup of the elements of finite order, Ann. of Math., 37 (1936), 766781.

3. Wu-chung Hsiang, Abelian groups characterized by their independent subsets, Pacific J. Math., 8 (1958), 447-457.

4. A. G. Kurosh, Theory of Groups, English trans. Vol. 1.

5. H. Prüfer, Untersuchungen über der Zerlegbarkeit der abzählbaren primaren abelschen. Gruppen, Math. Zeit., 17 (1923), 35-61. 


\title{
PACIFIC JOURNAL OF MATHEMATICS
}

\author{
EDITORS
}

\author{
Ralph S. Phillips \\ Stanford University \\ Stanford, California \\ F. H. Brownell \\ University of Washington \\ Seattle 5, Washington
}

\author{
A. L. Whiteman \\ University of Southern California \\ Los Angeles 7, California \\ L. J. PAige \\ University of California \\ Los Angeles 24, California
}

ASSOCIATE EDITORS
E. F. BECKENBACH
D. DERRY
H. L. ROYDEN
E. G. STRAUS
T. M. CHERRY
M. OHTSUKA
E. SPANIER
F. WOLF

\section{SUPPORTING INSTITUTIONS}

\author{
UNIVERSITY OF BRITISH COLUMBIA \\ CALIFORNIA INSTITUTE OF TECHNOLOGY \\ UNIVERSITY OF CALIFORNIA \\ MONTANA STATE UNIVERSITY \\ UNIVERSITY OF NEVADA \\ NEW MEXICO STATE UNIVERSITY \\ OREGON STATE COLLEGE \\ UNIVERSITY OF OREGON \\ OSAKA UNIVERSITY \\ UNIVERSITY OF SOUTHERN CALIFORNIA
}

\author{
STANFORD UNIVERSITY \\ UNIVERSITY OF TOKYO \\ UNIVERSITY OF UTAH \\ WASHINGTON STATE COLLEGE \\ UNIVERSITY OF WASHINGTON \\ * * * * \\ AMERICAN MATHEMATICAL SOCIETY \\ CALIFORNIA RESEARCH CORPORATION \\ HUGHES AIRCRAFT COMPANY \\ SPACE TECHNOLOGY LABORATORIES \\ NAVAL ORDNANCE TEST STATION
}

Mathematical papers intended for publication in the Pacific Journal of Mathematics should be typewritten (double spaced), and the author should keep a complete copy. Manuscripts may be sent to any one of the four editors. All other communications to the editors should be addressed to the managing editor, L. J. Paige at the University of California, Los Angeles 24, California.

50 reprints per author of each article are furnished free of charge; additional copies may be obtained at cost in multiples of 50 .

The Pacific Journal of Mathematics is published quarterly, in March, June, September, and December. The price per volume (4 numbers) is $\$ 12.00$; single issues, $\$ 3.50$. Back numbers are available. Special price to individual faculty members of supporting institutions and to individual members of the American Mathematical Society: $\$ 4.00$ per volume; single issues, $\$ 1.25$.

Subscriptions, orders for back numbers, and changes of address should be sent to Pacific Journal of Mathematics, 103 Highland Boulevard, Berkeley 8, California.

Printed at Kokusai Bunken Insatsusha (International Academic Printing Co., Ltd.), No. 6, 2-chome, Fujimi-cho, Chiyoda-ku, Tokyo, Japan.

\section{PUBLISHED BY PACIFIC JOURNAL OF MATHEMATICS, A NON-PROFIT CORPORATION}

The Supporting Institutions listed above contribute to the cost of publication of this Journal, but they are not owners or publishers and have no responsibility for its content or policies. 


\section{Pacific Journal of Mathematics}

\section{Vol. 11, No. 2 December, 1961}

Tsuyoshi Andô, Convergent sequences of finitely additive measures........

Richard Arens, The analytic-functional calculus in commutative topological algebras..........................................

Michel L. Balinski, On the graph structure of convex polyhedra in

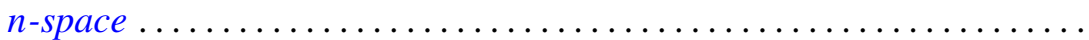

R. H. Bing, Tame Cantor sets in $E^{3}$...

Cecil Edmund Burgess, Collections and sequences of continua in the plane.

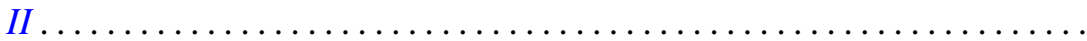

J. H. Case, Another 1-dimensional homogeneous continuum which contains an $\operatorname{arc}$

Lester Eli Dubins, On plane curves with curvature ................. 471

A. M. Duguid, Feasible flows and possible connections .............. 483

Lincoln Kearney Durst, Exceptional real Lucas sequences ................ 489

Gertrude I. Heller, On certain non-linear opeartors and partial differential equations........................................

Calvin Virgil Holmes, Automorphisms of monomial groups

Wu-Chung Hsiang and Wu-Yi Hsiang, Those abelian groups characterized by their completely decomposable subgroups of finite rank ..........

Bert Hubbard, Bounds for eigenvalues of the free and fixed membrane by finite difference methods .........................

D. H. Hyers, Transformations with bounded mth differences. .

Richard Eugene Isaac, Some generalizations of Doeblin's decomposition

John Rolfe Isbell, Uniform neighborhood retracts ..........

Jack Carl Kiefer, On large deviations of the empiric D. F. of vector chance variables and a law of the iterated logarithm...

Marvin Isadore Knopp, Construction of a class of modular functions and forms. II. . .

Gunter Lumer and R. S. Phillips, Dissipative operators in a Banach space....

Nathaniel F. G. Martin, Lebesgue density as a set function ...

Shu-Teh Chen Moy, Generalizations of Shannon-McMillan theorem ...

Lucien W. Neustadt, The moment problem and weak convergence in $L^{2}$

Kenneth Allen Ross, The structure of certain measure algebras...

James F. Smith and P. P. Saworotnow, On some classes of scalar-product algebras.

Dale E. Varberg, On equivalence of Gaussian measures. . 